\title{
Interplay between breathing and polar instabilities in transition metal perovskites with active A-sites
}

\author{
Atanu Paul, ${ }^{1}$ Anamitra Mukherjee, ${ }^{2}$ Indra Dasgupta, ${ }^{1}$ and Tanusri Saha-Dasgupta $\odot^{3, *}$ \\ ${ }^{1}$ School of Physical Sciences, Indian Association for the Cultivation of Science, Kolkata 700 032, India \\ ${ }^{2}$ School of Physical Sciences, National Institute of Science Education and Research, HBNI, Jatni 752050, India \\ ${ }^{3}$ Department of Condensed Matter Physics and Materials Science, S. N. Bose National Centre for Basic Sciences, Kolkata 700098, India
}

(Received 21 November 2019; accepted 25 February 2020; published 18 March 2020)

\begin{abstract}
Active A-site cations like $\mathrm{Bi}$ or $\mathrm{Pb}$ in $\mathrm{ABO}_{3}$ perovskites are known for their valence-skipping nature as well as for the stereochemical activities connected to lone pairs. While the former gives rise to breathing distortions in compounds like $\mathrm{BiNiO}_{3}$ or $\mathrm{PbCrO}_{3}$, the latter has been held responsible for ferroelectric, polar distortions in compounds like $\mathrm{BiFeO}_{3}$ or $\mathrm{PbVO}_{3}$. The microscopic origins of both distortions have been argued to stem from the hybridization between $\mathrm{Bi}(\mathrm{Pb})$ and $\mathrm{O}$. Employing first-principles calculations, together with a variational solution of the first-principles-inspired model Hamiltonian, we investigate the interplay of two types of distortion instabilities in $\mathrm{Bi} / \mathrm{Pb}$-based transition metal perovskites. Our study reveals that in the absence of orbital degeneracy of the $\mathrm{B}$ site, the preference of one over the other is dictated by the relative positioning of $\mathrm{O}$ $2 p$ level with respect to the A-site $6 p$ level. Closeness of the two levels favors polar distortion over the breathing and vice versa, level positioning of $\mathrm{O} 2 p$ being dictated by the strength of B-O hybridization.
\end{abstract}

DOI: 10.1103/PhysRevResearch.2.013333

\section{INTRODUCTION}

The physical properties [1] of transition metal (TM) perovskites of general formula $\mathrm{ABO}_{3}$, are believed to be dictated by the B-site TM $d$ electrons and the $\mathrm{O} p$ electrons. A-sites play the passive role of spectators, providing cohesion in the structure, introducing the size effect through a tolerance factor and ensuring charge neutrality. This conventional viewpoint gets modified when the $\mathrm{A}$-sites are occupied by cations like $\mathrm{Bi}$ or $\mathrm{Pb}$. As an example, $\mathrm{BiNiO}_{3}$ at ambient condition exhibits insulating behavior [2], while $\mathrm{LaNiO}_{3}$, with $\mathrm{La}^{3+}$ having a similar size as $\mathrm{Bi}^{3+}$, is a metal [3]. This is suggestive of the active role of $\mathrm{Bi}$ compared to the passive role of $\mathrm{La}$. This curious behavior has been recently explained [4] in terms of strong hybridization between extended $6 s$ orbital of $\mathrm{Bi}$ and $\mathrm{O}$ $2 p$ orbital, forming a ligand hole at the $\mathrm{O}$ site, that permits a $\mathrm{Bi}-\mathrm{O}$ breathing mode instability and resultant checkerboard pattern of compressed and expanded $\mathrm{BiO}_{12}$ polyhedra [cf. Fig. 1(a)]. The Bi cation in compressed polyhedra is associated with two holes $\left(\mathrm{Bi}^{3+} \underline{L}^{2}\right)$ and the $\mathrm{Bi}$ cation in expanded polyhedra has a negligible hole component, thereby fixing the occupancy of Ni $e_{g}$ states at half-filling and driving the Mott insulating state. On the other hand, with the valence configuration of $\mathrm{Bi}$ being $6 s^{2} 6 p^{3}$, the formally trivalent $\mathrm{Bi}$ in $\mathrm{ABO}_{3}$ perovskite ideally contains a lone pair of $6 s^{2}$ electrons. The $6 s^{2}$ lone pair has been implicated for the ferroelectric properties [5] of $\mathrm{BiFeO}_{3}$ [6] or $\mathrm{BiCoO}_{3}$ [7], through their

\footnotetext{
*t.sahadasgupta@gmail.com

Published by the American Physical Society under the terms of the Creative Commons Attribution 4.0 International license. Further distribution of this work must maintain attribution to the author(s) and the published article's title, journal citation, and DOI.
}

stereochemical activity, which manifests itself in off-centric polar movement of the A-cation within the $\mathrm{AO}_{12}$ cage [cf. Fig. 1(b)]. Microscopic origin of this phenomena has been also traced to strong Bi-O hybridization $[8,9]$.

This makes the situation curious. Since the driving mechanisms of both distortions appear to be propelled by the $\mathrm{Bi}-\mathrm{O}$ hybridization effect, it provokes the question whether there exists a common platform describing both distortions or whether there are important differences. Interestingly, a parallel almost exists between $\mathrm{Bi}$ and $\mathrm{Pb}$ compounds; $\mathrm{PbCrO}_{3}$ shows breathing distortion of alternate expansion and compression of $\mathrm{PbO}_{12}$ polyhedra [10], while compounds like $\mathrm{PbVO}_{3}$ or $\mathrm{PbTiO}_{3}$ show ferroelectric distortions [11,12].

In this communication, we investigate this intriguing scenario within the framework of density functional theory (DFT), along with solution of model Hamiltonian, constructed based on DFT essentials. This important problem is difficult due to various influencing factors. Given the complexity of the problem, the paper has been organized in the following manner. First, the trend of $\mathrm{O} 2 p$ level position with respect to A-site $6 s$ and $6 p$ positions is demonstrated in terms of band centers, on site energies, Wannier functions, and crystal orbital Hamiltonian population, considering four compounds: $\mathrm{BiFeO}_{3}, \mathrm{BiNiO}_{3}, \mathrm{PbVO}_{3}$, and $\mathrm{PbCrO}_{3}$. This tendency toward polar instability versus the breathing instability can be described within a common framework of A-site $6 s$ and $6 p$ orbitals hybridizing with $\mathrm{O} 2 p$ orbitals. The breathing versus polar instability is decided by the relative positioning of $6 \mathrm{~s}$ versus $6 p$ with respect to $\mathrm{O} 2 p$ states. As shown, this trend is strongly manifested for $\mathrm{BiFeO}_{3}$, and $\mathrm{BiNiO}_{3}$ and less pronounced for $\mathrm{PbVO}_{3}$ and $\mathrm{PbCrO}_{3}$. Second, the role of $\mathrm{B}$ site $(\mathrm{Fe}$ in $\mathrm{BiFeO}_{3}$ versus $\mathrm{Ni}$ in $\mathrm{BiNiO}_{3}$ ) to tune the $\mathrm{O} 2 p$ level position is discussed. Against this backdrop, with DFT essentials, a model Hamiltonian calculation is carried out which confirms the trend discussed on the basis of DFT results. This scenario, 
(a)

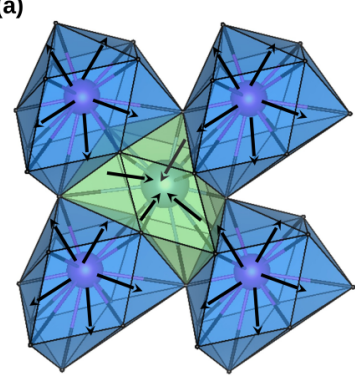

(b)

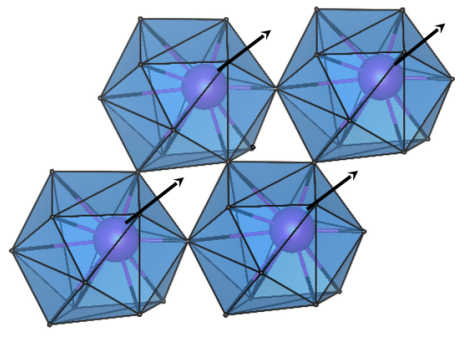

FIG. 1. (a) Breathing distortion of $\mathrm{AO}_{12}$ polyhedra in compounds like $\mathrm{BiNiO}_{3}$ or $\mathrm{PbCrO}_{3}$, exhibiting alternately expanded (marked in blue [dark gray]) and compressed (marked in light green [light gray]) $\mathrm{AO}_{12}$ polyhedra. (b) Off-centric, polar distortion of A cations within the $\mathrm{AO}_{12}$ cage of ferroelectric compounds like $\mathrm{BiFeO}_{3}$, along the specified direction of polarization. The B-site atoms have been omitted from the pictures for clarity.

however, is complex for perovskites, which have B-sites with orbital degeneracy, in which case the off-centric movement of the B-site, in addition to the A-site, contributes to polar distortions, and disentangling the two is difficult. We close the paper with discussion on such compounds. Our study paves a way to understanding an important yet difficult problem which needs future attention.

\section{NON-SPIN-POLARIZED ELECTRONIC STRUCTURE}

We start our discussion with DFT non-spin-polarized density of states (DOS). The DFT electronic structure calculations have been carried out in pseudopotential plane-wave basis with generalized gradient approximation (GGA) [13] and projected augmented wave potentials (PAW) [14] as implemented within the Vienna ab initio Simulation Package (VASP) [15]. The results have been validated in terms of all-electron augmented plane-wave calculations [16]; see the Supplemental Material (SM) for details [17].

The DOS projected to $\mathrm{Bi}(\mathrm{Pb}) 6 s, 6 p$, and $\mathrm{O} 2 p$ characters of $\mathrm{BiFeO}_{3}$ (BFO), $\mathrm{BiNiO}_{3}$ (BNO), $\mathrm{PbVO}_{3}$ (PVO), and $\mathrm{PbCrO}_{3}$ (PCO), at their respective undistorted phases, are shown in Figs. 2(a) and 2(b). The undistorted phases of BNO [2] and those of PVO [18] and PCO [10] are undisputed to be orthorhombic and cubic, respectively. However, the symmetry of the high-temperature nonpolar phase of BFO is controversial (cf. Fig. 1 in Ref. [19]), with cubic $(P m-3 m)$ [20], monoclinic $\left(P 2_{1} / m\right)$ [21], orthorhombic (Pbnm) [22], as well as centrosymmetric rhombohedral $(R-3 c)$ [23] symmetries suggested as different possibilities. While most results reported in the following [Figs. 2 and 3(a)] for BFO have been carried out assuming the cubic symmetry for the high-temperature phase, we have also carried out calculations considering the monoclinic, orthorhombic, and rhombohedral symmetries [17]. Some of the these results are included in the relevant figures [Fig. 3(b)].

The band centers and bandwidths of the respective bands, predominantly contributed by $\mathrm{Bi}(\mathrm{Pb}) 6 s, 6 p$ and $\mathrm{O} 2 p$ characters, extracted from the DOS plots, are shown in Fig. 2(c). The large mass of $\mathrm{Bi}$ and $\mathrm{Pb}$ separates the energies of $6 s$ and $6 p$ by several eVs, about $11 \mathrm{eV}$ for $\mathrm{Bi}$ and $10 \mathrm{eV}$ for $\mathrm{Pb}$. As is

(a)

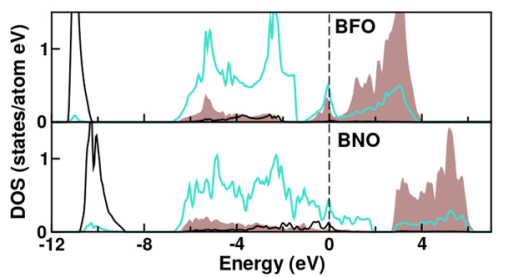

(b)
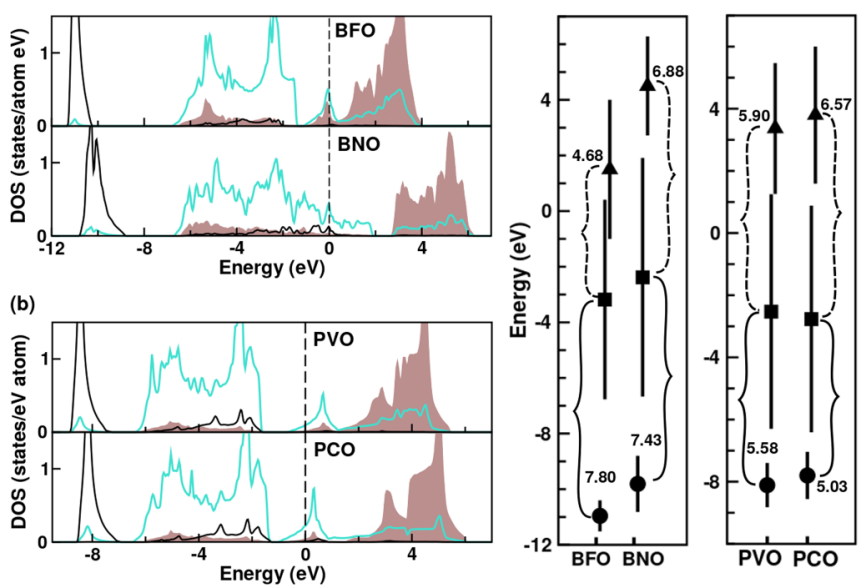

FIG. 2. (a) Non-spin-polarized density of states of BFO and $\mathrm{BNO}$, projected onto A $6 s$ (black), O $2 p$ (cyan [gray]), and $\mathrm{A}$ $6 p$ (brown [gray shaded]) characters. The zero of the energy is set at Fermi level. (b) Same as panel (a), but shown for PVO and PCO. (c) The band centers (A $6 s$, circles; O $2 p$, squares; A $6 p$, triangles) and band widths (vertical lines) of A $6 s, \mathrm{O} 2 p$, and $\mathrm{A} 6 p$ of $\mathrm{BFO}, \mathrm{BNO}, \mathrm{PVO}$, and PCO. The brackets show the differences of band centers between A-sites $6 s$ and $6 p$ and O-site $2 p$.

(a)

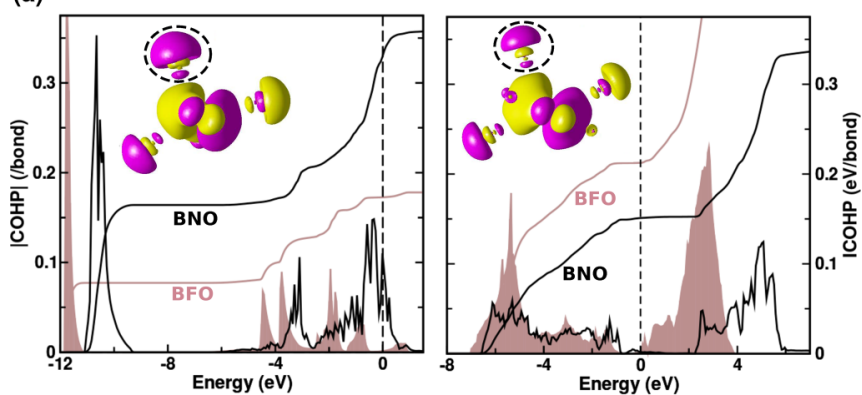

(b)

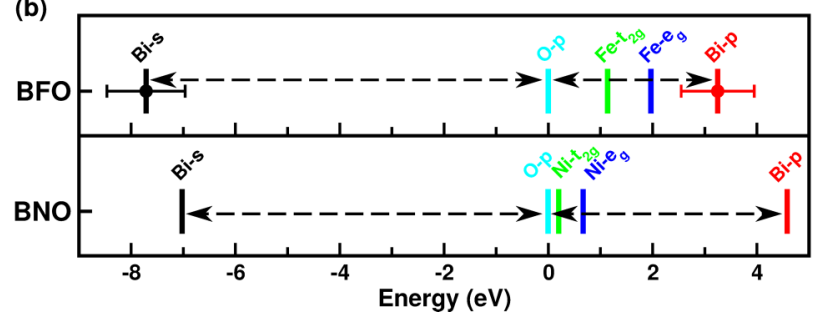

FIG. 3. (a) Absolute value of $\mathrm{Bi} 6 s-\mathrm{O} 2 p \mathrm{COHP}$ (left axis) and its cumulative sum, ICOHP (right axis), plotted as a function of energy, with zero of energy set at Fermi level for BNO (black) and BFO (brown [gray]). (b) Same as panel (a), but plotted for Bi $6 p$-O $2 p$. The values of COHP and ICOHP in panel (b) have been divided by three, to make them comparable with that of $\mathrm{Bi} 6 s-\mathrm{O} 2 p$. Insets show the constant amplitude surface of O- $p$ Wannier functions for BNO (left inset) and BFO (right inset). Yellow (light gray) and magenta (dark gray) colors indicate opposite signs. The weights at Bi sites are encircled. (c) Calculated energy level positions of $\mathrm{Bi}-s, \mathrm{O}-p$, and $\mathrm{B}(\mathrm{Ni} / \mathrm{Fe})-d$ in BNO and BFO. The zero of the energy is set at the average on-site energy of $\mathrm{O} p$. 
seen for $\mathrm{BFO}$, the primarily empty $\mathrm{Bi} 6 p$ states are close to $\mathrm{O}$ $2 p$ bands, strongly hybridizing and overlapping the respective bandwidths, while localized $\mathrm{Bi} 6 s$ states are situated about $11 \mathrm{eV}$ below Fermi energy $\left(E_{F}\right)$. Replacing $\mathrm{Fe}$ by $\mathrm{Ni}$ at B-site moves the $\mathrm{Bi} 6 s$ state closer to $\mathrm{O} 2 p$ states by a $\mathrm{eV}$ or so, while the $\mathrm{Bi} 6 p$ states gets pushed away from $\mathrm{O} 2 p$ states by couple of $\mathrm{eV}$, significantly weakening the $\mathrm{Bi} 6 p-\mathrm{O} 2 p$ admixture. A similar trend is observed between PVO and PCO, though less pronounced. As judged by the positions of band centers, the $\mathrm{Bi}(\mathrm{Pb}) 6 s$ state is moved closer to $\mathrm{O} 2 p$ states by about $0.5(0.5) \mathrm{eV}$ in the case of $\mathrm{BNO}$ (PCO) compared to $\mathrm{BFO}$ ( $\mathrm{PVO})$, while the $\mathrm{Bi}(\mathrm{Pb}) 6 p$ states in $\mathrm{BNO}(\mathrm{PCO})$ are moved away from $\mathrm{O} 2 p$ states by about $2.0(0.7) \mathrm{eV}$, compared to that in $\mathrm{BFO}(\mathrm{PVO})$. We thus conclude that while $\mathrm{Bi} / \mathrm{Pb} 6 p-\mathrm{O}$ $2 p$ covalency contributes in a significant manner in $\mathrm{Bi} / \mathrm{Pb}-\mathrm{O}$ hybridization in $\mathrm{BFO}$ or $\mathrm{PVO}$ compounds, i.e., those showing polar distortions, it is relatively weaker in compounds like $\mathrm{BNO}$ or PCO, i.e., those exhibiting breathing distortion. The hybridization in the latter cases is mostly governed by $\mathrm{Bi} / \mathrm{Pb}$ $6 s-\mathrm{O} 2 p$ covalency. As a passing comment, the change in hybridization is primarily governed by the change in on-site energies, with the change in hopping integrals being less important. The O- $p$ to Bi- $p$ energy separation between $\mathrm{BNO}$ and $\mathrm{BCO}$ changes by about $2.2 \mathrm{eV}$, which is about $50 \%$, while the $\mathrm{Bi}-\mathrm{O}$ bond length changes only by $1 \%$, resulting in a change in Bi $6 p-\mathrm{O} 2 p$ hopping $\left(t_{p p \sigma}\right)$ of only $1.05 \%$. In the paper, we thus focus primarily on the on-site energy differences. It is to be further noted that although the above analysis considers non-spin-polarized electronic structure results, inclusion of magnetism at the B site through spin-polarized calculations is found to keep the trend intact, as presented in the Supplemental Material. This confirms the robustness of the trend.

In order to ascertain the nature of A-O covalency on a quantitative level, we computed crystal orbital Hamiltonian population (COHP), which provides energy-resolved analysis of hybridization between a pair of atoms [24]. Figure 3(a) shows $\mathrm{Bi} 6 s-\mathrm{O} 2 p$ COHP in the left panel and $\mathrm{Bi} 6 p-\mathrm{O} 2 p$ COHP in the right panel, for $\mathrm{BNO}$ in comparison to that for BFO. We plot the absolute magnitudes of COHP, since we are primarily interested in the strength of the covalency. Integrated COHP (ICOHP) shows that its value at Fermi level $\left(E_{F}\right)$ for $\mathrm{Bi} 6 s-\mathrm{O} 2 p$ covalency is about two times larger in $\mathrm{BNO}$ than in $\mathrm{BFO}$, while for $\mathrm{Bi} 6 p-\mathrm{O} 2 p$ covalency it is about 0.65 times smaller in BNO compared to BFO. A similar trend in COHP/ICOHP between PCO and PVO is found (see the Supplemental Material), though less pronounced. The weakening of $6 p-2 p$ hybridization in BNO compared to $\mathrm{BFO}$ is further evident in the plots of Wannier functions in oxygen p-only Hamiltonian for BNO (left inset, Fig. 3) and BFO (right inset, Fig. 3), constructed using $N$ th-order muffin-tin orbital (NMTO) downfolding technique [17,25,26] by retaining only $\mathrm{O}-p$ degrees of freedom in the basis, with the rest including $\mathrm{Ni} / \mathrm{Fe}-d$ and $\mathrm{Bi}-s, p$ degrees of freedom being downfolded. As is seen from the plots, while the central part of the Wannier functions of oxygen $p$-only basis is shaped according to oxygen $p$ symmetry, the tails extending to $\mathrm{Ni} / \mathrm{Fe}$ and $\mathrm{Bi}$ are shaped according to integrated out $\mathrm{Ni} / \mathrm{Fe}-d$ and Bi degrees of freedom, reflecting the covalency. Significant amount of weights of the tails are found at the Bi sites (see encircled parts). $\mathrm{Bi} 6 s-\mathrm{O} 2 p$ mixed state further bonds to the empty $\mathrm{Bi} 6 p$ state, lowering the energy of the system, as is seen in the presence of $\mathrm{Bi} 6 p$-like shapes in the tails at Bi sites. Interestingly, the $6 p$ character in the Bi tail is weak in BNO, being more of $\mathrm{Bi} 6 s$, while the contribution of $\mathrm{Bi} 6 p$ character is relatively stronger in BFO (estimated to be $15 \%$ in $\mathrm{BNO}$ compared to $30 \%$ in $\mathrm{BFO}$ ). As argued by Watson et al. [9], the stereochemical activity of lone pairs is driven by the important contribution of mixing with $\mathrm{Bi} / \mathrm{Pb} 6 p$ states. The $\mathrm{Bi} 6 s-\mathrm{O} p$ interaction does not lead to energetic gain since both $\mathrm{Bi} 6 s$ and $\mathrm{O} 2 p$ are positioned below $E_{F}$. Energy gain happens through hybridization of these states with unoccupied $6 p$ states, which effectively moves $\mathrm{O} 2 p$, positioned at $E_{F}$ due to $\mathrm{Bi} 6 s-\mathrm{O} p$ interaction, away from $E_{F}$. In the event of weakening the $6 p$ contribution, as in the case of BNO, the contribution of Bi $6 s-\mathrm{O} 2 p$ mixed state at $E_{F}$ leads to formation of ligand hole, triggering the disproportionate charge-assisted breathing distortion of the A-O lattice [4].

Another useful way of looking at the stereochemical activity of the lone pair is considering its degree of localization around the atomic framework. Higher localization corresponds to higher asymmetry, which results in division of O-coordination around the A-site into two coordination spheres, as discussed in Ref. [27]. In order to estimate the degree of localization of the lone pair in a quantitative manner, we computed the spread of effective $\mathrm{Pb}$ or $\mathrm{Bi} 6 s$ Wannier function, obtained in a massive downfolding calculation by integrating out everything else other than A-site $s$. By construction, this takes into account the hybridization between $\mathrm{A}$ $6 s$, A $6 p$, and $\mathrm{O} 2 p$. The spread of such effective orbital for BFO is found to be about $22-27 \%$ smaller than that of BNO, suggestive of the localized and directional nature of the lone pair in BFO compared to the delocalized and symmetric one in BNO. See the Supplemental Material.

\section{ROLE OF B-SITE}

What decides the positioning of $\mathrm{O} 2 p$ within the the $\mathrm{Bi} / \mathrm{Pb}$ $6 s$-6p energy window? Figure 3(b) shows the Bi-s, TM- $d$, and O- $p$ energy level positions in BFO averaged over possible nonpolar phases in comparison to those of $\mathrm{BNO}$, obtained from the tight-binding representation of the Hamiltonian in the $\mathrm{Bi} 6 s p, \mathrm{Ni} / \mathrm{Fe} 3 d$, and $\mathrm{O} 2 p$ basis within the NMTOdownfolding calculations $[25,28]$. As is seen, moving across the third row of the periodic table from $\mathrm{Fe}$ to $\mathrm{Ni}$, the TM $d$ energy level position gets closer to the oxygen $p$ energy level, resulting in stronger $d-p$ interaction between the B-site and oxygen in BNO compared to BFO. The bonding oxygen $p$ level, renormalized by the TM $d$-O $p$ hybridization, gets pushed down, thus being positioned farther from $\mathrm{Bi} p$ in BNO compared to BFO. The position of the oxygen $p$ level, being tuned by $\mathrm{B}$-site-O hybridization, thus decides the fate of the active A-site, exhibiting breathing or polar distortion.

\section{MODEL STUDY}

In order to capture the competition between the two instabilities within a general framework, we considered variational calculation of a DFT-inspired model Hamiltonian, consisting of A-site $s, p$, and $\mathrm{O} p$. Interestingly enough, it was found that competition between breathing and polar distortion at the 
A sublattice can be captured without active involvement of $\mathrm{B}$-sites. The B-site $d$ orbitals, responsible for the insulating nature (Mott or dimerized) of the compounds, have frozen charge fluctuations. Therefore, they drop out out of the problem, being included in an implicit manner and dictating the position of $\mathrm{O} p$ through tuning of $\mathrm{B}-\mathrm{O}$ hybridization. The following convention is adopted. A-site coordinate is chosen as $\Delta \equiv(1 / 2,1 / 2,1 / 2)$, with origin set at the B-site. $\eta$ 's refer to oxygen positions around a given A-site. The effect of the distortion in bond lengths is incorporated through the Harrison scaling of the hopping elements [29], while the directional nature of the $s-p$ and $p-p$ hopping is captured using appropriate Slater-Koster integrals in the hopping elements. The model Hamiltonian can be written as a sum of three terms: (i) on-site term $H_{1}$, (ii) hopping term $H_{2}$, and (iii) restoring force term for polar and breathing distortions $\mathrm{H}_{3}$ :

$$
\begin{aligned}
H_{1}= & \sum_{\mathbf{r}, \sigma, \alpha} \varepsilon_{A, \alpha} b_{\mathbf{r}+\Delta, \alpha, \sigma}^{\dagger} b_{\mathbf{r}+\Delta, \alpha, \sigma} \\
& +\sum_{\mathbf{r}, \sigma, \alpha} \varepsilon_{O x, \alpha} s_{\mathbf{r}+\Delta+\eta, \alpha, \sigma}^{\dagger} s_{\mathbf{r}+\Delta+\eta, \alpha, \sigma}, \\
H_{2}= & -t^{A-O} \sum_{\mathbf{r}, \eta, \alpha, \beta, \sigma}\left(M_{\alpha, \beta}^{\eta} b_{\mathbf{r}+\Delta, \alpha, \sigma}^{\dagger} s_{\mathbf{r}+\Delta+\eta, \beta, \sigma}+\text { H.c. }\right), \\
H_{3}= & \frac{1}{2} \kappa_{B} \sum_{\mathbf{r}, \eta, \alpha, \beta} \delta_{B}^{2}+\frac{1}{2} \kappa_{P} \sum_{\mathbf{r}, \eta, \alpha, \beta} \delta_{P}^{2}
\end{aligned}
$$

with

$$
M_{\alpha, \beta}^{\eta}=g_{\alpha, \beta}\left[1+f_{\alpha, \beta}^{\eta} \delta_{B}(-1)^{x+y+z}+p_{\alpha, \beta}^{\eta} \delta_{P}(-1)^{q(\mathbf{r})}\right],
$$

where $b_{r, \alpha, \sigma}^{\dagger}$ and $s_{r, \alpha, \sigma}^{\dagger}$ create an electron in the orbital $\alpha(s, p)$ at the A-site and in the $p$ orbital at an oxygen atom located at $\mathbf{r}$, respectively, and spin is denoted by $\sigma$. The A-site and the oxygen on-site energies are denoted by $\varepsilon_{A, \alpha}$ and $\varepsilon_{O x, \alpha}$ respectively. $t^{A-O}$ sets the overall energy scale while the prefactor $g_{\alpha, \beta}$ in $M_{\alpha, \beta}^{\eta}$ contains the symmetry dependency of the hopping. Breathing and polar distortions are captured in terms of modulation of hopping via distortion amplitudes $\delta_{B}$ and $\delta_{P}$ respectively. $f_{\alpha, \beta}^{\eta}$ and $p_{\alpha, \beta}^{\eta}$ encode the orbital-dependent Harrison scaling factors and the long and short bond effect resulting from polar distortion, respectively. $\kappa_{B}$ and $\kappa_{P}$ refer to spring constants for $\delta_{B}$ and $\delta_{P}$, respectively. For uniform polar distortion, we choose $q(\mathbf{r})=0$ and along the (111) direction. Based on DFT estimates and previous literature [4], we choose $t_{s p}^{A-O} \approx t_{p p \sigma}^{A-O}=1.5 \mathrm{eV}, t_{p p \pi}^{A-O}=-0.3 \mathrm{eV}$, setting the on-site energies for $\varepsilon_{A, s}$ and $\varepsilon_{A, p}$ to be zero and $11 \mathrm{eV}$ respectively. We vary $\kappa_{B} / \kappa_{P}$ as well as $\varepsilon_{O x}$. We stress that $\delta_{B}$ and $\delta_{P}$ are variational parameters that induce phase competition between distortions of two distinct symmetries. Thus, a phase transition occurs when the ground-state energies with the two kinds of distortions cross each other as a function of $\varepsilon_{O x}$ and $\kappa_{B} / \kappa_{P}$. In this sense, one can identify $\delta_{B}$ and $\delta_{P}$ as indicators of phase transition.

The phase diagram of breathing and polar distorted phases in the $\kappa_{B} / \kappa_{P}$ versus $\varepsilon_{O x}$ (scaled by $t_{p p \sigma}$ ) plane is shown in Fig. 4(a). The phase boundary is constructed by following the evolution of $\delta_{B}$ and $\delta_{P}$, as a function of $\varepsilon_{O x}$, for every ratio of $\kappa_{B} / \kappa_{P}$ explored here. Typical data, for realistic DFT estimates of $\kappa_{B} / \kappa_{P}=1$, are shown in Fig. 3(b) and exhibit strong first-
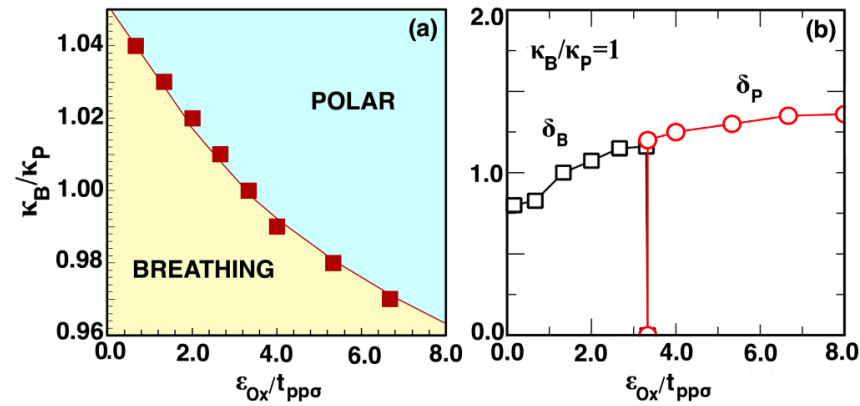

FIG. 4. (a) Model phase diagram in $\kappa_{B} / \kappa_{P}-\varepsilon_{\text {Ox }}$ plane. The regions in the parameter space showing polar and breathing instability have been marked. The solid line is a guide to the eye. (b) The evolution of the breathing $\left(\delta_{B}\right)$ and polar $\left(\delta_{P}\right)$ distortions amplitudes as a function of $\varepsilon_{\mathrm{Ox}}$, for $\kappa_{B} / \kappa_{P}=1$.

order transition as $\varepsilon_{O x} / t_{p p \sigma}$ crosses 3.33 or $\varepsilon_{O x}=5 \mathrm{eV}$, from a breathing mode to a polar mode phase. We find that the phase transition remains first order for all other $\kappa_{B} / \kappa_{P}$ ratios shown in the phase diagram. This phenomenology is in good agreement with conclusions drawn from DFT calculations [cf. Fig. 2(c)], proving the robustness and generality of our conclusions.

\section{COMPOUNDS WITH ASSOCIATED ORBITAL DEGENERACIES AT B-SITE}

In our discussion so far, we focused on lattice instabilities arising out of only the A-site. However, the situation gets complex when the lattice instabilities associated with orbital degeneracies at the $\mathrm{B}$-site also participate. A pertinent example in this context is $\mathrm{BiCoO}_{3}(\mathrm{BCO})$. The $\mathrm{B}$-site TM element, $\mathrm{Co}$, lies in between $\mathrm{Fe}$ and $\mathrm{Ni}$ in the periodic table. The calculated energy-level diagram in $\mathrm{BCO}$ shows the positioning of $\mathrm{Bi} 6 \mathrm{~s}$ and $\mathrm{Bi} 6 p$, relative to $\mathrm{O} 2 p$, to be more similar to that of $\mathrm{BNO}$ than that of BFO (see the Supplemental Material). This would support suppression of polar instability and promotion of breathing instability. Calculated spread of Bi $s$ effective Wannier function also supports this idea. However, BCO is found to be ferroelectric with polar, tetragonal crystal structure [7]. This polar distortion, however, is primarily driven by the $d^{6}$ configuration of high-spin configuration $\mathrm{Co}^{3+}$ which prefers a large off-centric displacement of the $\mathrm{Co}^{3+}$. This situation may be compared to the case of PVO, which in its polar, tetragonal phase shows the off-centric vanadyl distortion of $\mathrm{V}^{4+}$ with $d^{1}$ occupancy. As pointed out before, the difference between $\mathrm{PVO}$ and $\mathrm{PCO}$ is much less obvious than BFO and BNO, making the situation indeed similar to that of $\mathrm{BCO}$ [30].

In this respect, $\mathrm{BiCrO}_{3}$ with $\mathrm{Cr}$ in its orbitally inactive $d^{3}$ configuration is more akin to $\mathrm{BFO}$, which according to phase diagram presented in Fig. 4, should show polar instability. As was found experimentally [31] as well as theoretically [32], $\mathrm{BiCrO}_{3}$, like its counterpart $\mathrm{BiFeO}_{3}$, is unstable in the ideal cubic perovskite phase, with a structural instability driven by the stereochemical activity of the $\mathrm{Bi}$ lone pair. Following the same guideline, one would expect $\mathrm{PbFeO}_{3}$ with orbitally inactive Fe $d^{5}$ nominal state to show a disproportionate chargedriven breathing distortion at the A-site, as in the case of PCO. Such a suggestion was indeed made [33], and $\mathrm{PbFeO}_{3}$ was 
synthesized under high pressure [34], but the situation remains to be settled.

\section{CONCLUSION}

In summary, analyzing the first-principles electronic structure, combined with model Hamiltonian study, we examine the instability toward polar versus breathing distortions of $\mathrm{Bi} / \mathrm{Pb}-\mathrm{O}$ sublattice in TM perovskites. Our study reveals that instability toward breathing or polar instability is dictated by relative mixing of $\mathrm{O} p$ states with filled A-site $s$ state and empty A-site $p$ states. Choice of B-site tunes the position of effective $\mathrm{O} p$ states by influencing the $\mathrm{B}-\mathrm{O}$ hybridization.
Replacement of $\mathrm{Fe}$ by $\mathrm{Ni}$ at the $\mathrm{B}$-site of $\mathrm{BiFeO}_{3}$ thus triggers breathing distortion instead of polar. This general picture may get modified in the presence of orbital degeneracy at the B-site, which can lead to polar distortion of B-O sublattice, dominating over the instabilities at A-O sublattice.

\section{ACKNOWLEDGMENTS}

T.S.-D. thanks the Department of Science and Technology, India, for the support through Thematic Unit of Excellence. I.D. thanks the Department of Science and Technology, India, for support.
[1] D. Khomskii, Transition Metal Compounds (Cambridge University Press, Cambridge, UK, 2014).

[2] S. Ishiwata, M. Azuma, M. Takano, E. Nishibori, M. Takata, M. Sakata, and K. Kato, J. Mater. Chem. 12, 3733 (2002).

[3] A. Sakai, G. Zheng, and Y. Kitaoka, J. Phys. Soc. Jpn. 71, 166 (2002).

[4] A. Paul, A. Mukherjee, I. Dasgupta, A. Paramekanti, and T. Saha-Dasgupta, Phys. Rev. Lett. 122, 016404 (2019).

[5] T. Atou, H. Chiba, K. Ohoyama, Y. Yamaguchi, and Y. Syono, J. Solid State Chem 145, 639 (1999).

[6] W. Kaczmarek and Z. Pajak, Solid State Commun. 17, 807 (1975).

[7] K. Oka, M. Azuma, W. Chen, H. Yusa, A. A. Belik, E. Takayama-Muromachi, M. Mizumaki, N. Ishimatsu, N. Hiraoka, M. Tsujimoto, M. G. Tucker, J. Attfield, and Y. Shimakawa, J. Am. Chem. Soc. 132, 9438 (2010).

[8] G. W. Watson and S. C. Parker, J. Phys. Chem. B 103, 1258 (1999).

[9] G. W. Watson, S. C. Parker, and G. Kresse, Phys. Rev. B 59, 8481 (1999).

[10] R. Yu, H. Hojo, T. Watanuki, M. Mizumaki, T. Mizokawa, K. Oka, H. Kim, A. Machida, K. Sakaki, Y. Nakamura et al., J. Am. Chem. Soc. 137, 12719 (2015).

[11] A. Belik, M. Azuma, T. Saito, Y. Shimakawa, and M. Takano, Chem. Mater. 17, 269 (2005).

[12] G. Shirane and S. Hoshino, J. Phys. Soc. Jpn. 6, 265 (1951).

[13] J. P. Perdew, K. Burke, and M. Ernzerhof, Phys. Rev. Lett. 77, 3865 (1996).

[14] P. E. Blöchl, Phys. Rev. B 50, 17953 (1994).

[15] G. Kresse and J. Furthmuller, Phys. Rev. B 54, 11169 (1996).

[16] P. Blaha, K. Schwarz, G. K. H. Madsen, D. Kvasnicka, J. Luitz, R. Laskowski, F. Tran, and L. D. Marks, WIEN2K, an Augmented Plane Wave + Local Orbitals Program for Calculating Crystal Properties (Karlheinz Schwarz, Techn. Universität Wien, Austria, 2018).

[17] See Supplemental Material at http://link.aps.org/supplemental/ 10.1103/PhysRevResearch.2.013333 for details of calculations, the crystal structures, the results for $\mathrm{BCO}$, spin-polarized results, COHP for PVO and PCO, the computed spreads, and details related to the model study.
[18] A. A. Belik, T. Yamauchi, H. Ueda, Y. Ueda, H. Yusa, N. Hirao, and M. Azuma, J. Phys. Soc. Jpn. 83, 074711 (2014).

[19] J. Wei, C. Wu, T. Yang, Z. Lv, Z. Xu, D. Wang, R. Haumont, and Z. Cheng, J. Phys. Chem. C 123, 4457 (2019).

[20] R. Palai, R. S. Katiyar, H. Schmid, P. Tissot, S. J. Clark, J. Robertson, S. A. T. Redfern, G. Catalan, and J. F. Scott, Phys. Rev. B 77, 014110 (2008).

[21] R. Haumont, I. A. Kornev, S. Lisenkov, L. Bellaiche, J. Kreisel, and B. Dkhil, Phys. Rev. B 78, 134108 (2008).

[22] D. C. Arnold, K. S. Knight, F. D. Morrison, and P. Lightfoot, Phys. Rev. Lett. 102, 027602 (2009).

[23] S. M. Selbach, T. Tybell, M.-A. Einarsrud, and T. Grande, Adv. Mater. 20, 3692 (2008).

[24] R. Dronskowski and P. E. Blöchl, J. Phys. Chem. 97, 8617 (1993); F. Boucher and R. Rousseau, Inorg. Chem. 37, 2351 (1998).

[25] O. K. Andersen and T. Saha-Dasgupta, Phys. Rev. B 62, R16219(R) (2000).

[26] O. K. Andersen and O. Jepsen, Phys. Rev. Lett. 53, 2571 (1984).

[27] L. M. Volkova and D. V. Marinin, J. Supercond. Nov. Magn. 24, 2161 (2011)

[28] For BFO, the energy level positions of $\mathrm{Bi} 6 s$ and $6 p$ are found to vary among the various suggested high-temperature phases. Thus, a mean position and the rms deviation of the positions considering different suggested crystal structures (see the Supplemental Material) are shown.

[29] W. A. Harrison, Electronic Structure and Properties of Solids (Freeman, San Francisco, 1980).

[30] The $d^{0}$-ness at the B-site also may contribute in deciding the distortion. An example is $\mathrm{PbTiO}_{3}$, where the stereochemical activity of the lone pair of $\mathrm{Pd}^{2+}$ and ferroelectric $\mathrm{Ti}^{4+}$ displacement interact to give rise to the polar, tetragonal structure.

[31] M. Murakami, S. Fujino, S.-H. Lim, C. J. Long, L. G. Salamanca-Riba, M. Wuttig, I. Takeuchi, V. Nagarajan, and A. Varatharajan, Appl. Phys. Lett. 88, 152902 (2006).

[32] N. Hill, P. Battig, and C. Daul, J. Phys. Chem. B 106, 3383 (2002).

[33] J. B. Goodenough and J. Zhou, Sci. Technol. Adv. Mater. 16, 036003 (2015).

[34] T. Tsuchiya, H. Saito, M. Yoshida, T. Katsumata, T. Ohba, Y. Inaguma, T. Tsurui, and M. Shikano, Mater. Res. Soc. Symp. Proc. 988, QQ09 (2007). 